\title{
How to speak at international conferences
}

\author{
V Wright
}

Medical conferences with multinational audiences are commonplace, so that many of us will have to speak to audiences for whom English is not their first language. Our traditional insular arrogance, coupled with a defective education system, make it unlikely that we will take the trouble to become fluent in a tongue other than English, and even if we did, Japanese, Urdu, and Serbo-Croatian are likely to elude us. Some simple rules are, therefore, useful in addressing foreign audiences. The advice given in an article on 'A short course on biomechanics for Japanese surgeons' ${ }^{\prime}$ is relevant and should be heeded.

I have definite views on how overseas talks should be done, and have checked out my prejudices on 84 overseas doctors from 20 countries. These colleagues were given a questionnaire containing $10 \mathrm{~cm}$ visual analogue scales, ranging from unimportant to very important, for each of the following preconceived ideas-to speak slowly, to use short sentences, to avoid jokes, to use slides, to restrict the number of slides.

\section{Speak slowly and distinctly}

The first overseas conference I attended was in Rome. An American was waxing eloquent on the metabolism of corticosteroids in rheumatic patients. His speed was that of an express train, and his profusion of biochemical terms overwhelmed his medical audience, let alone the distraught translators. Agonising pleas to slow down were disregarded. The simultaneous translators gave up, and the hall was filled with the click, click from the machines of exasperated delegates who concluded that the electronic wizardry had capitulated; it was not so-just the translators throwing in the towel. Speaking slowly was the second most important feature recognised by the respondents to the questionnaire, scoring a mean of $6 \cdot 5$.

\section{Use short, complete sentences}

The construction of sentences in another language may require the translator to hear the total before starting. This is not easy if the sentence expands to a paragraph before a full stop is reached. For the same reason, if the translation is consecutive, do not break off in the middle of a sentence. It is worth remembering that symbols may be difficult to translate. I was speaking in Addis Ababa when I quoted Einstein's formula $E=m^{2}$. Only afterwards did I learn that Amharic has no vowels. The translator coped with the situation by saying,
'You heard him; you heard him'. When the translation is simultaneous, pauses after a few sentences help the translator to catch up, the audience to assimilate your talk, and you to gather your thoughts.

The use of short sentences is valued by overseas doctors. Their mean score on this point was $6 \cdot 2$. The additional appended comments were of interest. Idioms should be avoided, as should abbreviations. Speakers were urged to speak English English, not American English. English was spoken most clearly by Germans, Swiss, and Swedes! Presumably that was because they had a more limited vocabulary and spoke more slowly than the equivalent Englishman.

\section{Do not tell jokes}

Funny stories seldom translate into another culture. The humour from a quick-fire American comedian, which doubles up his American audience with laughter, but leaves an English audience bemused or shell-shocked, should teach us that. Some jokes which a European audience find acceptable, may even be distasteful and objectionable to another nationality. These represent my prejudices. Although shared by many overseas doctors, this reaction was not universal. Indeed, the mean score for avoiding them was only $3 \cdot 3$. One respondent even added, 'It is important to joke, especially in the jolly British way'.

Give the translator a manuscript beforehand You may not stick exactly to the text, but it is helpful to the translators to know the terrain through which you are passing.

\section{Use slides wisely and well}

The use of slides was far and away the most important in the minds of respondents with a mean score of $9 \cdot 4$, and few scored below 9 . Clinical slides were particularly valued. The main points should be on a slide, and a conclusion slide should be constructed. The writing on the slides should, if possible, be in basic English. A good picture is worth a hundred words in any language. The principles of slide construction have been discussed. ${ }^{2}$ The article should be read and put into practice.

The mean score for restriction of the number of slides was $5 \cdot 2$. Do not show too many slides. A good rule of thumb is one slide per minute. If you think you may have too many slides, you have.
Accepted for publication 17 June 1992
Rehabilitation Research Unit, University of Leeds Leeds LS2 9NZ, United Kingdom 
Keep to time

Because you are speaking more slowly, you will say less. A quart cannot be poured into a pint pot. Should the chairman reduce your time, do not speak faster; bite your tongue, restrain your exasperation, and cut your coat according to your cloth. Do not tell the chairman what you think of him until your travelling expenses have been paid.

If you are chairing a symposium at an international conference, I would recommend that you give all speakers a copy or a synopsis of this article. That should include the nationalsat a recent conference in Madrid I found the local Spaniards were the cruelest to the translators in terms of their speed of delivery. It may not do much to bring the participants to heel, but at least it will give you a clear conscience in that you have done what you could, and it may improve your own performance.

1 Seedhom B B, Smeathers J E, Thompson D T. A shor course on biomechanics for Japanese surgeons. BMF 1985 291: 802-4.

2 Evans M. Use slides. In: How to do it. London: British Medical Association, 1979: 103-7. 